\title{
Ethnic Difference of Disease Prevalence in Rural China: Examples and Explanations
}

\author{
Ming Guan1,2 \\ ${ }^{1}$ School of Management, Xuchang University, Xuchang, China \\ ${ }^{2}$ Family Issues Center, Xuchang University, Xuchang, China \\ Email: gming0604@sohu.com
}

Received 14 March 2015; accepted 13 April 2015; published 15 April 2015

Copyright (c) 2015 by author and Scientific Research Publishing Inc.

This work is licensed under the Creative Commons Attribution International License (CC BY). http://creativecommons. org/licenses/by/4.0/

(c) (i) Open Access

\begin{abstract}
Ethnic difference of disease prevalence has attracted great attentions in recent years in China, but few researches have summarized analysis available on ethnic difference of disease prevalence in rural China. The PubMed Central, Wiley Inter science, Science direct, Biomed central, CNKI and Springer-link were searched to identify studies published between January 1984 and October 2014 on ethnic inequality of health status in rural China. Distinct ethnic differences of disease prevalence exist in rural China. Results across disciplines put different explanations on the ethnic differences from ethnicity, infant feeding, and inequality in maternal health services utilization angles. The ethnic inequality of health status in rural China can be reduced by policy makers to allocate more resources towards health service in ethnic rural China.
\end{abstract}

\section{Keywords}

Ethnic Difference, Rural China, Disease Prevalence

\section{Introduction}

There are 55 nationally recognized minorities in China, comprising just less than $9 \%$ of China's total population. The five largest ethnic minority groups are Zhuang, Manchu, Moslems or Hui, Miao, and Uygur or Weiwu'er. Chinese minority groups are mainly distributed in the mountain area. In the 1950s, five provinces with large minority populations were designated as autonomous minority nationality regions: Xinjiang, Inner-Mongolia, Tibet, Ningxia, and Guangxi. Genetic backgrounds, customs, culture, and food consumption among ethnic minority groups are different. In the term of the health status of Chinese ethnic minorities, using the China Health and Nutrition Survey data collected during 1989-2006, Ouyang and Pinstrup-Andersen (2012) found negative and significant differences between minority and Han Chinese in a set of anthropometric measures for people of 
all age groups [1].

Here, prior studies are collected to address the insight and understanding of the ethnic difference of disease prevalence in rural China. It also aims to describe knowledge and practices of the disease prevention in ethnic rural China.

\section{Methods}

\subsection{Aim}

To systematically identify and review literature related to ethnic difference of disease prevalence in rural China in order to determine the extent of previous research efforts, current knowledge about the prevalence and nature in this population.

\subsection{Search Question}

What is the current level of knowledge in the literature concerning ethnic difference of disease prevalence in rural China?

\subsection{Search Strategy}

The search strategy is systematically applied to available databases of published literature online. The database searches and the Internet searches were undertaken in November 2014.

\subsection{Databases}

Computerised bibliographical databases that were accessible through the Xuchang University library were searched without any year restriction. The databases searched include PubMed Central, Wiley Inter science, Science direct, Biomed central, CNKI and Springer-link.

\subsection{Key Words}

The key words/phrases used in the search of databases and Internet sites include the following:

Health state AND rural China.

Health inequality AND China.

Health AND ethnic China.

Disease prevalence.

\subsection{Study Selection}

Very broad criteria for consideration of studies were applied:

Participants: a participant cohort that included ethnic Chinese people of any age.

Interventions: all intervention models included.

Outcome measures: all outcome measures included.

Study design: all designs (both qualitative and quantitative research) were included as well as reviews, abstracts, letters, media releases and published reports.

\subsection{Exclusion Criteria}

Studies of Han ethnic peoples from in China.

Those papers those were not accessible through the Xuchang University libraries.

Non-English language studies.

\section{Results}

The number and level of evidence of peer-reviewed papers and published ethnic differences of disease prevalence is outlined. See Table 1 . The search strategy identified 9 peer-reviewed descriptive studies in the past 10 years. It is clear that the available literature is largely low level evidence, in line with the nature of previous research efforts. 
Table 1. Ethnic differences of disease prevalence.

\begin{tabular}{ccc}
\hline Author & Size, ethnicity, age of study & Findings \\
& population, and location
\end{tabular}

Leung and Chu (2003) [2]

Dental caries and periodontal status

Child behavior problems

$\begin{array}{cc}\text { Li, et al. } & \begin{array}{c}\text { Child behavior } \\ \text { problems }\end{array} \\ \text { (2001) [3] } & \\ & \\ \text { Li, et al. } & \text { Protein-energy } \\ \text { (1999) [4] } & \text { malnutrition }\end{array}$

Hotta, et al. The risk factors for (2005) [5] low Kaup index

He, et al. (2014) [6]

General Obesity and Abdominal Obesity

Zhang, et al. (2013) [7]

Hypertension

Cai, et al.

(2011) [8]

Diabetes

Zhao, et al. (2010) [9]
196 12-year-old (118 Han and 78 from ethnic minorities, i.e. Muslims and Tibetans) in 3 primary schools in Hexi Townships, Hainan Tibetan Autonomous State, Qinghai Province

1222 rural mother-child pairs belonging to Hani, Yi, Hui, Miao and Han in 4 economically disadvantaged minority counties in Yunnan Province

2019 children below 7 years belonging to ethnic minority groups and the Han major group in 4 poor rural minority counties in Yunnan Province

1173 mother-child pairs in 36 villages of the Dai peoples' autonomous region in Yunnan Province

8036 nomadic Kazakhs and rural Uyghur residents ( $\geq 18$ years old) in 18 villages, Xinjiang, China, about $4407 \mathrm{~km}$ away from capital Beijing

2133 Bai ethnic adults aged 50 or above in rural communities in Dali, southwest China

2939 ethnic minorities individuals aged 18 and over in rural Yunnan province

1612 rural original adults in rural Ningxia
The prevalence of caries experience in Han children is significantly lower than that in Muslim and Tibetan children in rural Qinghai. Dental caries and caries treatment needs of Han and minority rural schoolchildren in Qinghai are low but their periodontal health status is unsatisfactory. Basic dental health care and preventive education for the children in this part of China is needed.

The child behavior problems were prevalent in children aged 2 - 6 years in rural minority children of China. Ineffective family child-rearing practices increase risk for child behavior problems.

Protein-energy malnutrition is relatively high in the rural minority children of China. Chronic socioeconomic underdevelopment and genetic effects, rather than a severe or immediate lack of food, may lead to protein-energy malnutrition.

The mother's child-rearing behavior and social support were identified as risk factors. An offer of effective and abundant information to mothers, and the existence of professionals with whom mothers can consult immediately, were required to construct a better health system in the target area.

Both general and abdominal obesity were common in rural ethnic Kazakhs and Uyghurs. The prevalence rates were different in these two minorities depending on ethnicity, gender, and age. Kazakhs, females and elderly people may be prioritized in prevention of obesity in western China.

Hypertension prevalence is high among the population of Bai ethnic group in China, while the associated risk factors of hypertension include overweight/obesity, cigarette smoking, history of hypertension, and older age. The percentages of hypertensive participants aware of their hypertension and those taking antihypertensive medications were low with an incredibly low proportion of hypertensive patients who kept their hypertension under control.

Among diabetic respondents, those belonging to an ethnic minority had a lower probability of being aware they suffered from diabetes and being treated with antidiabetic medication than Han Chinese.

The ethnic-specific prevalence of the metabolic syndrome was $10.3 \%$ in Han ethnic group and $13.7 \%$ in Hui ethnic group. Components of metabolic syndrome and metabolic syndrome were more common in Hui ethnic group than Han ethnic group in rural residents' adults in Ningxia. 
Nguyen, et al (2014) [10]

Hypertension (HTN) and type 2 diabetes mellitus (DM)
11 villages randomly selected from Yunnan province
Gao, et al. (2011) [11]

\section{Overweight and obesity}

1255 subjects aged 20 - 75 years
in Liangshan Yi Autonomous
Prefecture of Sichuan province
The prevalence of DM was 6.3\%, with variability between ethnic groups, ranging from $2.7 \%$ in the Tibetan group to $9.8 \%$ in the Han group $(\mathrm{P}=0.09)$. HTN prevalence also varied from $30.5 \%$ in the Lisu group to $54.7 \%$ in the Tibetan group $(\mathrm{P}=0.003)$. Only $22.5 \%$ (9/40) and $47.7 \%$ (112/235) of those diagnosed with DM and HTN, respectively, had reported a known history of their condition. The prevalence of DM varied across ethnic groups, with Han people having a greater prevalence than the ethnic minorities.

The prevalence of overweight/obesity in the $\mathrm{Yi}$ nationality is similar to that in Chinese adults 5 years ago. However, urban residents have a much higher prevalence of overweight/obesity than their rural counterparts.

Complementally, in a cross-sectional survey, Li, et al. (2012) found ethnic groups living in Xinjiang had striking differences in cardiovascular disease risk factors (CRFs; hypertension, obesity, diabetes, dyslipidemia, smoking) [12]. In Hainan, the prevalence of asthma in the Li ethnic group was 3.38\%, much higher than the national average level in China. Aging, agriculture industry (the rubber industry in particular), rural residence, family history of asthma, history of allergies, cold air, inhalation of dust and irritant gases, smoking, domestic cooking fuel and living environment were associated with increased risk of asthma [13]. The prevalence of dyslipidemia, diabetes, and current smoking among older Korean Chinese with hypertension were 75.4\%, 6.6\%, and $23.1 \%$ respectively [14].

In fact, not all diseases demonstrate ethnic difference. An investigation sponsored by Kunming Huaxia secondary specialized school in Yunnan confirmed there was no significant difference in deafness associated gene mutational spectrum and frequency between the Yunnan minority and Han patients [15]. Results from the review showed that there exist significant ethnic differences of disease prevalence in rural China. Next, the causes of ethnic differences need explanations.

\section{Explanations}

Ethnic differences of disease prevalence mainly may be due to inequality in social status as for gender disparities [16], inequality of access to health care services [17], spatial inequality in health care [18], differences in life expectancy [19], regional health inequality [20]. Ouyang and Pinstrup-Andersen (2012) suggest that the economic development level of the province of residence is a major factor contributing to the health gap between minority and Han Chinese [1].

Living environment and habits may be the second cause. Using data from the Chinese national survey on students’ physical fitness and health from 1985 to 2005, Ma, et al. (2009) find the physical functions and the athletic ability of the minority students in China had the advantages and characteristics, which might be related to their living environment and habits [21]. A cross-sectional survey conducted in rural areas of Yunnan Province, China from 2010 to 2011 find individuals belonging to an ethnic minority had a lower probability of exposure to secondhand smoke and nicotine dependence [22]. Social selection and the costs of relative deprivation appear to be useful to the understanding of health inequality in rural China, though in a manner shaped by the particular social context [23]. Chen and Meltzer (2008) suggest that, among rural Chinese residents, increasing community average income and income inequality are positively associated with both obesity and hypertension [24].

The third cause may be different responses to drugs from different ethnic groups in China. In an open label, parallel-group study, healthy volunteers (10 Hans, 10 Mongolians, 10 Uygurs, 10 Huis and 9 Koreans) of Chinese nationality received a single oral tablet dose of $15 \mathrm{mg}$ midazolam. The results are midazolam maximum concentration $(\mathrm{C}(\max ))$ was significantly lower in Mongolians than that in Hans, Uygurs, Huis and Koreans $(74.9 \pm 33.7,103.1 \pm 26.4,124.8 \pm 50.0,130.0 \pm 38.3$ and $189.0 \pm 82.1 \mu \mathrm{g} / \mathrm{L}$, respectively). Midazolam terminal half-life $(\mathrm{t}(1 / 2 \mathrm{z}))$ were $3.0 \pm 0.8,2.2 \pm 0.7,1.9 \pm 0.7,3.5 \pm 1.9$, $3.8 \pm 2.3 \mathrm{~h}$ for Hans, Mongolians, Uygurs, Huis and Koreans, respectively [25]. 
The fourth cause may be ethnic factors. Innate factor is mainly concerned with heredity and race. A study aimed to explore the association between HLA-DRB1 alleles and anti-neutrophil cytoplasmic antibodies among Uyghur and Han patients with ulcerative colitis in China finds Genetic polymorphisms of the HLA-DRB $1 * 08$ and *13 may contribute to the clinical heterogeneity of ulcerative colitis between Uyghur and Han ulcerative colitis patients in China. In Uyghur ulcerative colitis patients, HLA-DRB1*13 may be correlated with anti-neutrophil cytoplasmic antibodies positivity [26]. The gene frequencies of ADH2(2) and ALDH2(2) were lower in Tibetan and Mongolian populations than in Vietnamese, Han Chinese, and three Chinese minority populations [27].

The fifth cause may be infant feeding. A longitudinal study of infant feeding practices with 1219 mothers (578 Han, 360 Uygur and 281 "other minority" mothers) report "exclusive breastfeeding" rates in the Han, Uygur and "other minority" groups at discharge were $78.0 \%, 34.5 \%$ and $83.1 \%$ respectively, at six months they had fallen to $4.8 \%, 0.4 \%$ and $16.8 \%$ respectively [28]. Breastfeeding is speculated to influence later-life health of an infant.

The sixthcause may be health literacy. An in-person interviews with 913 rural women aged 23 - 57 (57.5\% Hui minorities/42.5\% Han ethnicity) in Ningxia poor minority area revealed Hui minorities had 1.65 times higher rates of low health literacy and 1.22 and 1.25 times for pain/discomfort and anxiety/depression impairments, respectively. Low health literacy was associated with poor HRQoL, with a $23 \%$ increase in the prevalence of pain/discomfort impairments after adjusting for socio-demographics. This association was significant in the Hui group $(\mathrm{PR}=1.30,95 \% \mathrm{CI}=1.06-1.58)$ but not for the Han group $(\mathrm{PR}=0.99,95 \% \mathrm{CI}=0.76-1.30)$ [29].

The final cause may be inequality in maternal health services utilization. A cross-sectional study from 10 provinces in rural Western China in 2005 reported that Han ethnicity woman, particularly in conjunction with high school education and rich wealth status, was the main contributor to inequality in maternal health services utilization. And it suggests that an effective way to reduce the inequality is not only to narrow the gap of income between the rich and poor, but focus education on ethnic minority woman in rural remote areas [30].

\section{Conclusions}

The research effort undertaken aims to date on ethnic differences of disease prevalence in rural China. The ethnic differences of disease prevalence in China were produced due to socio-economic, ethnic, human, and policymaking causes. The key point is inequality distribution of health resources and poor primary health care services in rural and remote mountains.

The prevalence of diseases is high among ethnic minorities in rural China, representing an important health problem. More effort should be devoted to the study, prevention, and treatment of these diseases. Effective strategies are needed to reduce the incidence of diseases in this ethnic population. Public health should be more concerned with social policies and social determinants than with health services and disease control. Governments should make sure that high-quality health service can reach remote ethnic rural China. Zheng, et al. (2011) suggest policy-makers should attempt to improve economic development in less developed regions and to improve health policies and the public health systems that address the needs of everyone [19]. I also agree with Fang, et al. (2010) that investment in health resources can help China achieve and maintain equality in distribution of health [20]. In fact, primary health care plays a more important role than hospital services in reducing ethnic disparities in health. Strategies that can enhance public awareness of diseases and increase access to affordable medications are urgently needed, especially for poor, less educated individuals who belong to ethnic minorities. Ethnic-specific strategies should be developed to prevent disease in different ethnic groups, as ethnic villagers with diseases were not actually aware of their condition.

\section{Acknowledgements}

This study was funded by the "Wellbeing and quality of life of minority families migrating from rural to urban areas" sponsored by the Ministry of Education of China Humanities and Social Sciences Research Youth Project Fund (Project number:14YJC630042).

\section{References}

[1] Ouyang, Y. and Pinstrup-Andersen, P. (2012) Health Inequality between Ethnic Minority and Han Populations in Chi- 
na. World Development, 40, 1452-1468. http://dx.doi.org/10.1016/j.worlddev.2012.03.016

[2] Leung, W.K. and Chu, C.H. (2003) Dental Caries and Periodontal Status of 12-Year-Old School Children in Rural Qinghai, China. International Dental Journal, 53, 73-78. http://dx.doi.org/10.1111/j.1875-595X.2003.tb00662.x

[3] Li, Y., Shi, A., Wan, Y., Hotta, M. and Ushijima, H. (2001) Child Behavior Problems: Prevalence and Correlates in Rural Minority Areas of China. Pediatrics International, 43, 651-661. http://dx.doi.org/10.1046/j.1442-200X.2001.01446.X

[4] Li, Y., Guo, G., Shi, A., Li, Y., Anme, T. and Ushijima, H. (1999) Prevalence and Correlates of Malnutrition among Children in Rural Minority Areas of China. Pediatrics International, 41, 549-556. http://dx.doi.org/10.1046/j.1442-200x.1999.01103.x

[5] Hotta, M., Li, Y., Anme, T. and Ushijima, H. (2005) Risk Factors for Low Kaup Index among Children in Rural Ethnic Minority Areas of Yunnan, China. Pediatrics International, 47, 147-153. http://dx.doi.org/10.1111/j.1442-200x.2005.02045.x

[6] He, J., Guo, S., Liu, J., Zhang, M., Ding, Y., et al. (2014) Ethnic Differences in Prevalence of General Obesity and Abdominal Obesity among Low-Income Rural Kazakh and Uyghur Adults in Far Western China and Implications in Preventive Public Health. PLoS ONE, 9, e106723.

[7] Zhang, J., Huang, Q., Yu, M., Cha, X., Li, J., et al. (2013) Prevalence, Awareness, Medication, Control, and Risk Factors Associated with Hypertension in Bai Ethnic Group in Rural China: The Yunnan Minority Eye Study. PLoS ONE, 8, e70886. http://dx.doi.org/10.1371/journal.pone.0070886

[8] Cai, L., Dong, J., Shu, Z., Lu, Y. and Tao, J. (2011) Socioeconomic Differences in Diabetes Prevalence, Awareness, and Treatment in Rural Southwest China. Tropical Medicine and International Health, 16, 1070-1076. http://dx.doi.org/10.1111/j.1365-3156.2011.02805.x

[9] Zhao, Y., Jin, J., Liu, X., Xu, H., Yang, J. and Zhang, Y. (2010) Prevalence of the Metabolic Syndrome among rural Original Adults in NingXia, China. BMC Public Health, 10, 140.

[10] Nguyen, T.-L.N., Khalife, S.Z., Tang, S.Y., Hu, D., Huo, Y., Dustin, L., Azen, S.P. and Detrano, R.C. (2014) Prevalence of Diabetes and Hypertension in Ethnic Minority Adults Living in Rural Yunnan Province. China. International Journal of Diabetes in Developing Countries, 34, 50-55. http://dx.doi.org/10.1007/s13410-013-0137-Z

[11] Gao, Y., Ran, X.W., Xie, X.H., Lu, H.L., Chen, T., Ren, Y., Long, Y. and Tian, H.M. (2011) Prevalence of Overweight and Obesity among Chinese Yi Nationality: A Cross-Sectional Study. BMC Public Health, 11, 919. http://dx.doi.org/10.1186/1471-2458-11-919

[12] Li, N., Wang, H., Yan, Z., Yao, X., Hong, J. and Zhou, L. (2012) Ethnic Disparities in the Clustering of Risk Factors for Cardiovascular Disease among the Kazakh, Uygur, Mongolian and Han Populations of Xinjiang: A Cross-Sectional Study. BMC Public Health, 12, 499. http://dx.doi.org/10.1186/1471-2458-12-499

[13] Ding, Y.P., Yao, H.X., Tang, X.L., He, H.W., Shi, H.F., Lin, L., Li, M., Chen, S., Chen, J. and Wang, H.J. (2012) An Epidemiology Study of Bronchial Asthma in the Li Ethnic Group in China. Asian Pacific Journal of Tropical Medicine, 5, 157-161. http://dx.doi.org/10.1016/S1995-7645(12)60016-9

[14] Li, C.Y., Han, H.R., Kim, J. and Kim, M.T. (2011) Factors Related to Risk of Cardiovascular Disease among Older Korean Chinese with Hypertension. Asian Nursing Research, 5, 164-169. http://dx.doi.org/10.1016/j.anr.2011.09.002

[15] Xin, F., Yuan, Y., Deng, X., Han, M., Wang, G., Zhao, J., Gao, X., Liu, J., Yu, F., Han, D. and Dai, P. (2013) Genetic Mutations in Nonsyndromic Deafness Patients of Chinese Minority and Han Ethnicities in Yunnan, China. Journal of Translational Medicine, 11, 312. http://dx.doi.org/10.1186/1479-5876-11-312

[16] Yu, M.Y. and Sarri, R. (1997) Women’s Health Status and Gender Inequality in China. Social Science \& Medicine, 45, 1885-1898. http://dx.doi.org/10.1016/S0277-9536(97)00127-5

[17] Luo, J., Zhang, X., Jin, C. and Wang, D. (2009) Inequality of Access to Health Care among the Urban Elderly in Northwestern China. Health Policy, 93, 111-117. http://dx.doi.org/10.1016/j.healthpol.2009.06.003

[18] Zhang, X. and Kanbur, R. (2005) Spatial Inequality in Education and Health Care in China. China Economic Review, 16, 189-204. http://dx.doi.org/10.1016/j.chieco.2005.02.002

[19] Zheng, X., Song, X., Chen, G., You, Y., Ren, Q., Liu, J., Zhang, L., Tan, L., Wei, J. and Chen, Q. (2011) Health Inequalities during 20 Years of Rapid Economic Development in China (1980-2000): A Mortality Analysis. Biomedical and Environmental Sciences, 24, 329-334.

[20] Fang, P., Dong, S., Xiao, J., Liu, C., Feng, X. and Wang, Y. (2010) Regional Inequality in Health and Its Determinants: Evidence from China. Health Policy, 94, 14-25. http://dx.doi.org/10.1016/j.healthpol.2009.08.002

[21] Ma, J., Zhang, J., Wu, S.S., Song, Y., Hu, P.J. and Zhang, B. (2009) Changes of Physical Functions among Chinese Minority Students from 1985 to 2005. Zhonghua Liu Xing Bing Xue Za Zhi, 30, 1039-1042. [Article in Chinese].

[22] Cai, L., Wu, X., Goyal, A., Han, Y., Cui, W., Xiao, X., He, J., Zhao, K., Song, Y. and Jiao, F. (2012) Patterns and So- 
cioeconomic Influences of Tobacco Exposure in Tobacco Cultivating Rural Areas of Yunnan Province, China. BMC Public Health, 12, 842. http://dx.doi.org/10.1186/1471-2458-12-842

[23] Anson, O. and Sun, S. (2004) Health Inequalities in Rural China: Evidence from HeBei Province. Health \& Place, 10, 75-84. http://dx.doi.org/10.1016/S1353-8292(03)00048-0

[24] Chen, Z. and Meltzer, D. (2008) Beefing up with the Chans: Evidence for the Effects of Relative Income and Income Inequality on Health from the China Health and Nutrition Survey. Social Science \& Medicine, 66, 2206-2217. http://dx.doi.org/10.1016/j.socscimed.2008.01.016

[25] Guo, T., Mao, G.F., Xia, D.Y., Su, X.Y. and Zhao, L.S. (2011) Pharmacokinetics of Midazolam Tablet in Different Chinese Ethnic Groups. Journal of Clinical Pharmacy and Therapeutics, 36, 406-411. http://dx.doi.org/10.1111/j.1365-2710.2010.01178.x

[26] Gao, F., Aheman, A., Lu, J.J., Abuduhadeer, M., Li, Y.X. and Kuerbanjiang, A. (2014) Association of HLA-DRB1 Alleles and Anti-Neutrophil Cytoplasmic Antibodies in Han and Uyghur Patients with Ulcerative Colitis in China. Journal of Digestive Diseases, 15, 299-305. http://dx.doi.org/10.1111/1751-2980.12143

[27] Chen, S.H., Zhang, M., Wang, N.S. and Scott, C.R. (1994) Gene Frequencies of Alcohol Dehydrogenase $\left(\mathrm{ADH}_{2}\right)$ and Aldehyde Dehydrogenase ${ }_{2}\left(\mathrm{ALDH}_{2}\right)$ in Five Chinese Minorities. Human Genetics, 94, 571-572. http://dx.doi.org/10.1007/BF00211030

[28] Xu, F., Binns, C., Nazi, G., Shi, L., Zhao, Y. and Lee, A. (2006) A Comparison of Breastfeeding among Han, Uygur and Other Ethnic Groups in Xinjiang, PR China. BMC Public Health, 6, 196. http://dx.doi.org/10.1186/1471-2458-6-196

[29] Wang, C., Li, H., Li, L., Xu, D., Kane, R.L. and Meng, Q. (2013) Health Literacy and Ethnic Disparities in HealthRelated Quality of Life among Rural Women: Results from a Chinese Poor Minority Area. Health and Quality of Life Outcomes, 11, 153. http://dx.doi.org/10.1186/1477-7525-11-153

[30] Liu, X., Gao, W. and Yan, H. (2014) Measuring and Decomposing the Inequality of Maternal Health Services Utilization in Western Rural China. BMC Health Services Research, 14, 102. http://dx.doi.org/10.1186/1472-6963-14-102 